\title{
Correlación entre la estimación de la edad de gestación por palpación rectal y la edad de gestación real en la vaca
}

\section{Correlation between the estimation of the gestational age by rectal palpation and the actual gestation age in the cow}

\author{
González T, Marco ${ }^{1,2}$, M. Sc, Oviedo S, Teresa Ph.D, Vergara G, Oscar ${ }^{1}$ Ph.D.
}

${ }^{1}$ Universidad de Córdoba, Facultad de Medicina Veterinaria y Zootecnia.

Departamento de Ciencias Pecuarias. Montería, Colombia.

${ }^{2}$ Instituto de Investigaciones Biológicas del Trópico (IIBT).

\section{Keywords:}

Cow;

pregnancy diagnosis;

rectal palpation.

\section{Palabras Clave:}

Diagnóstico de gestación; palpación rectal; vaca.

\begin{abstract}
The correlation between the estimated time of gestation by rectal palpation and the actual gestation time in cattle was determined. Sixteen farms were selected and visited every 8-10 weeks to perform the gestation diagnoses. Data were obtained from 2,204 cows; 1,349 were commercial Zebu and 855 dual purpose. The gestation diagnoses were performed and by a single professional without knowledge of the dates of service. The minimum service time for the diagnosis of gestation by rectal palpation was 30 days, the maximum of 290 days. Pearson correlation was performed between the time estimated by rectal palpation and the real time of gestation of the animals. Pearson's correlation analysis showed that there is a high correlation between the estimation of gestational age by rectal palpation and the actual gestational age of the animals. A decrease in this correlation was also observed between 150-240 days and increased again after 240 days of gestation.
\end{abstract}

\section{Resumen}

Se determinó la correlación entre la estimación del tiempo de gestación por palpación rectal y el tiempo real de gestación en el ganado bovino. Se seleccionaron 16 fincas y se visitaron cada 8-10 semanas para realizar los diagnósticos de tiempo de gestación. Los datos se obtuvieron de 2.204 vacas; 1.349 fueron cebú comercial y 855 de doble propósito. Los diagnósticos de gestación fueron realizados y por un solo profesional sin conocimiento de las fechas de servicio. El tiempo mínimo de servicio para el diagnóstico de gestación por palpación rectal fue de 30 días, el máximo de 290 días. Se realizó correlación de Pearson entre el tiempo estimado por palpación rectal y el tiempo real de gestación de los animales. El análisis de correlación de Pearson demostró que existe alta correlación entre la estimación de la edad gestacional por palpación rectal y la edad gestacional real de los animales. También se observó una disminución de esta correlación entre 150-240 días y aumentó nuevamente después de los 240 días de gestación. 


\section{Introducción}

La determinación del tiempo de gestación en bovinos y por consiguiente el estado de fertilidad de un rebaño, es un factor zootécnico de mucha importancia para el manejo de un hato. El diagnóstico de gestación y tiempo de la misma, es una práctica que indirectamente aumenta la eficiencia productiva, porque facilita el manejo adecuado de las hembras en diferentes fases de gestación, contribuye con el cuidado preparto, durante el parto, en el postparto, así como también con el descarte de hembras con problemas reproductivos de imposible solución.

El estado de preñez en la vaca puede ser determinado por métodos clínicos y de laboratorio. Los clínicos incluyen efecto doppler, eco-pulsos ultrasónicos, radiografía, ecografía y palpación rectal. Los de laboratorio incluyen la determinación de hormonas, factor temprano de preñez, gonadotropinas, antígenos y biopsia vaginal, entre muchos otros. A pesar de la existencia de múltiples métodos, la palpación rectal del tracto reproductivo y su contenido es el método más utilizado en la vaca tanto para el diagnóstico de preñez, como para la estimación del tiempo de gestación y un sinnúmero de estados fisiológicos y patológicos que permiten tomar decisiones tanto al productor como al profesional.

La palpación rectal y la ecografía transrectal son dos métodos que permiten un diagnóstico inmediato de gestación en vacas (ROMANO et al 2006). Sin embargo, en la práctica, la palpación rectal continúa siendo el método más frecuentemente utilizado por el profesional veterinario de campo para realizar el diagnóstico de preñez (YOUNGQUIST 2006; WARNICK et al. 1995; MOMONT 1990.).

El objetivo de los métodos de diagnóstico de gestación es determinar con la mayor precisión posible que no haya falsos positivos o falsos negativos. Además, el método se debe aplicar lo más pronto posible después del servicio (monta natural, inseminación o trasferencia embrionaria) y adicionalmente se debe determinar la edad del embrión o feto (ZEMANJIS 1966; YOUNGQUIST 2006).

Diversos trabajos realizados sobre palpación rectal en bovinos, buscan correlacionar esta técnica con mortalidad embrionaria. Sin embargo, los resultados no son concluyentes y se afirma que la muerte fetal puede no ser una causa habitual de la palpación rectal temprana de vacas para diagnóstico la gestación. No obstante se requiere de mucho entrenamiento con el fin de desarrollar un alto nivel de destrezas manuales que le permitan al profesional realizar la manipulación correcta y delicada del tracto reproductivo (ABBITT et al 1978; VAILLANCOURT et al 1979; THURMOND y PICANSO 1993; YOUNGQUIST 2006).
Según WARNICK et al (1995), una de las situaciones que limitan el uso de la palpación para determinación de la gestación, es el intervalo relativamente largo entre el servicio y el tiempo en que un diagnóstico preciso pueda ser realizado. Aunque existen estudios que demuestran que la ecografía tiene una eficiencia superior para el diagnóstico temprano de gestación (27 días), con respecto a la palpación rectal, estos mismos trabajos, concluyen que son necesarios estudios de costo-beneficio para implementar esta técnica como práctica rutinaria en las fincas ganaderas. En este sentido se puede afirmar que la palpación rectal tiene la ventaja de requerir solo de un profesional experimentado y bien entrenado en la técnica y no de equipamientos costosos (THURMOND y PICANSO 1993; VAILLANCOURT et al 1979).

El objetivo del presente trabajo fue comparar la eficiencia de la técnica de la palpación rectal para estimar la edad gestacional en vacas de cría y de doble propósito.

\section{Materiales y Métodos}

Tipo de estudio. Se realizó un estudio descriptivo de corte transversal, en donde se examinaron hembras bovinas pertenecientes al municipio de Montería, Córdoba, Colombia.

Sitio del estudio. La zona agroecológica en donde se realizó el estudio se caracteriza por presentar una topografía semiondulada, con alturas que van desde los 13 hasta los $40 \mathrm{msnm}$. La precipitación anual alcanza los $1300 \mathrm{~mm}$, humedad relativa del $85 \%$ y la temperatura promedio anual es de $29^{\circ} \mathrm{C}$.

Fincas. Se seleccionaron 16 fincas ubicadas en el municipio de Montería a las cuales se les realizó una visita técnica cada 8-10 semanas con el fin de realizar los diagnósticos de tiempo de gestación.

Animales. Los datos para este estudio se obtuvieron de 2.204 vacas de las cuales, 1.349 fueron cebú comercial (alto mestizaje de Bos indicus) sin ordeño, (tipo carne) y 855 animales pertenecientes al sistema de doble propósito (ordeño una vez al día) que son animales cruzados entre Bos indicus x Bos taurus en distintas proporciones.

Técnica de palpación rectal. Se utilizó el método de palpación rectal descrito por ZEMANJIS 1966 y por YOUNGQUIST 2006, los cuales se basan fundamentalmente en la presencia de cuatro signos positivos de preñez, así como el tamaño de algunos de ellos (deslizamiento de membranas fetales, vesícula amniótica, feto y placentomas). 
Los diagnósticos de gestación fueron realizados por un solo profesional sin conocimiento de las fechas de servicio (monta natural o inseminación artificial). El tiempo mínimo de servicio para someter una hembra a diagnóstico de gestación por palpación rectal fue de 30 días, independiente de la aptitud (carne o doble propósito) de los animales. Con el fin de analizar los datos, estos fueron divididos en rangos de 30 días hasta el final de la gestación.

Confrontación de diagnóstico vs fecha real de servicio. Los datos obtenidos de diagnóstico de tiempo de gestación fueron registrados en tablas y confrontados posteriormente con las fechas de monta natural o inseminación anotados en los registros oficiales de las tarjetas o software de los animales de cada finca según el caso. Posteriormente se realizaron las correlaciones de la precisión del diagnóstico mediante palpación y el tiempo real de gestación según el servicio reportado o registrado.

Análisis estadístico. Los datos fueron sometidos a análisis de estadística descriptiva. Se realizó correlación de Pearson entre el tiempo estimado por palpación y el tiempo real de gestación de los animales de acuerdo con el servicio registrado en la hoja de vida de cada animal.

\section{Resultados}

El resumen de los resultados de las vacas tipo carne se muestra en la tabla 1 y para las de doble propósito en la tabla 2. El análisis de correlación de Pearson (Tabla 3), demostró que existe alta correlación entre la estimación de la edad gestacional por palpación rectal y la edad gestacional real de los animales. No obstante, se observó una disminución de esta correlación entre 150-240 días y aumentó después de los 240 días de gestación (Figura 1).

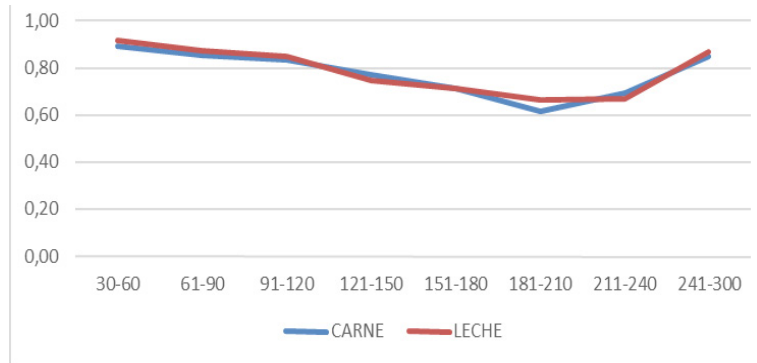

Figura 1. Correlación de Pearson entre el tiempo estimado por palpación y el tiempo real de gestación en ganado bovino.

Tabla 1. Media de las desviaciones del valor estimado por palpación con relación al tiempo real de gestación en ganado tipo carne.

\begin{tabular}{|c|c|c|c|c|}
\hline $\begin{array}{c}\text { Intervalo para el examen de } \\
\text { gestación (días) }\end{array}$ & $\mathrm{N}$ & Media de desviaciones & Desviación estándar & Error estándar \\
\hline $30-60$ & 416 & 0.4567 & 4.3468 & 0.2131 \\
\hline $61-90$ & 441 & -0.748 & 5.3921 & 0.2568 \\
\hline $91-120$ & 250 & -0.008 & 6.0963 & 0.3517 \\
\hline $121-150$ & 102 & -0.814 & 8.3456 & 0.7125 \\
\hline $151-180$ & 65 & -2.815 & 11.231 & 1.1521 \\
\hline $181-210$ & 32 & -3.188 & 14.518 & 1.9305 \\
\hline $211-240$ & 15 & -3.6 & 19.911 & 3.2599 \\
\hline $241-300$ & 28 & -8.25 & 10.956 & 1.5211 \\
\hline General & 1349 & -0.589 & 6.3062 & 0.1652 \\
\hline
\end{tabular}

Tabla 2. Media de las desviaciones del valor estimado por palpación con relación al tiempo real de gestación en ganado doble propósito.

\begin{tabular}{ccccc}
\hline $\begin{array}{c}\text { Intervalo para el examen de } \\
\text { gestación (días) }\end{array}$ & $\mathrm{N}$ & Media de desviaciones & Desviación estándar & Error estándar \\
\hline $30-60$ & 201 & -0.005 & 3.8778 & 0.2467 \\
$61-90$ & 206 & -1.641 & 5.4752 & 0.3446 \\
$91-120$ & 176 & -0.432 & 6.8353 & 0.4613 \\
$121-150$ & 100 & -1.42 & 9.4781 & 0.8159 \\
$151-180$ & 52 & -3.25 & 11.416 & 1.2766 \\
$181-210$ & 46 & -0.826 & 11.213 & 1.3126 \\
$211-240$ & 35 & -2.029 & 15.347 & 1.9799 \\
$241-300$ & 39 & -4.962 & 13.61 & 1.691 \\
\hline General & 855 & -1.047 & 7.0086 & 0.2283 \\
\hline
\end{tabular}


Tabla 3. Correlación de Pearson entre el tiempo estimado de gestación por palpación rectal y el tiempo real en ganado bovino.

\begin{tabular}{|c|c|c|c|c|c|c|}
\hline \multirow{2}{*}{$\begin{array}{l}\text { Intervalo para el examen de } \\
\text { gestación (días) }\end{array}$} & \multicolumn{2}{|c|}{ General } & \multicolumn{2}{|c|}{ Cebú } & \multicolumn{2}{|c|}{ Leche } \\
\hline & Correlación & $\mathrm{P}$ valor & Correlación & $P$ valor & Correlación & $\mathrm{P}$ valor \\
\hline $30-60$ & 0.89082 & $<.0001$ & 0.87559 & $<.0001$ & 0.91658 & $<.0001$ \\
\hline $61-90$ & 0.85212 & $<.0001$ & 0.84365 & $<.0001$ & 0.87267 & $<.0001$ \\
\hline $91-120$ & 0.83596 & $<.0001$ & 0.82178 & $<.0001$ & 0.84671 & $<.0001$ \\
\hline $121-150$ & 0.76892 & $<.0001$ & 0.79125 & $<.0001$ & 0.74837 & $<.0001$ \\
\hline $151-180$ & 0.71250 & $<.0001$ & 0.71266 & $<.0001$ & 0.71184 & $<.0001$ \\
\hline $181-210$ & 0.61500 & $<.0001$ & 0.55497 & 0.0010 & 0.66358 & $<.0001$ \\
\hline $211-240$ & 0.69250 & $<.0001$ & 0.75834 & 0.0011 & 0.66934 & $<.0001$ \\
\hline $241-300$ & 0.84802 & $<.0001$ & 0.82805 & $<.0001$ & 0.86665 & $<.0001$ \\
\hline General & 0.99312 & $<.0001$ & 0.99188 & $<.0001$ & 0.99396 & $<.0001$ \\
\hline
\end{tabular}

En la tabla 1 se muestran las diferencias entre el valor estimado del tiempo de gestación por palpación rectal y el valor real de la gestación. En las tablas 2 y 3 se presentan las medias de los diferentes rangos de edad entre el valor estimado de la edad del feto por palpación rectal y el valor real en ganado tipo carne y doble propósito respectivamente.

La tabla 3 muestra los coeficientes de correlación para los diferentes rangos de edad entre el valor estimado del tiempo de gestación por palpación rectal y el valor real de acuerdo con el servicio.

\section{Discusión}

La detección temprana de gestación en vacas se constituye en una herramienta fundamental que permite la rápida toma de decisiones encaminadas a disminuir los días abiertos y concomitantemente permite reducir las pérdidas económicas en explotaciones bovinas. Adicionalmente, el diagnóstico de gestación es un elemento clave en los programas de salud reproductiva del rebaño (OLTENACU et al., 1990) y paralelamente contribuye con el control de inventarios en cada finca cuando esta evaluación se realiza sistemática y técnicamente.

La palpación transrectal del útero es el método directo más antiguo y ampliamente utilizado para el diagnóstico temprano de gestación en vacas. La palpación transrectal de la vesícula amniótica fue descrita por WISNICKY Y CASSIDA en 1948, mientras que el deslizamiento de las membranas corioalantoideas entre el pulgar y el índice a los 30 días después de la gestación fue descrita por Zemjanis en 1970 (FRICKE et al. 2016)
Es incuestionable que la gestación en vacas se puede interrumpir por ruptura manual de la vesícula amniótica durante el proceso de palpación rectal (LÓPEZGATIUS, 2005); diversos estudios han investigado la magnitud de la pérdida de gestación iatrogénica inducida por palpación transrectal, sin embargo, los resultados son controversiales (THURMOND Y PICANSO, 1993), por lo que esta técnica para diagnóstico de gestación sigue vigente.

Los beneficios económicos del diagnóstico de preñez dependen de varios factores tales como la eficiencia en la detección del estro, el tiempo transcurrido después de la inseminación o monta hasta la realización del diagnóstico, la exactitud del diagnóstico, el efecto del diagnóstico por palpación transrectal sobre la pérdida embrionaria, entre otros. (OLTENACU et al 1990). Los resultados de este estudio confirman, en particular, la importancia de la alta cualificación del profesional en el diagnóstico preciso de la edad gestacional desde etapas tempranas de gestación, lo que repercute en disminución de pérdidas económicas y acelera la toma de decisiones tanto médicas como zootécnicas.

Los métodos directos para diagnóstico temprano de gestación implican la detección directa de estructuras asociadas al producto de la concepción; la especificidad y sensibilidad de la prueba dependen notablemente de la experiencia y competencia del profesional y el tiempo transcurrido después de la inseminación o monta hasta cuando se realice la técnica. Profesionales experimentados pueden lograr una alta sensibilidad y especificidad en el diagnóstico de gestación por palpación rectal en la especie bovina (FRICKE et al. 2016), como se observó en este estudio. 
Se encontró una alta correlación entre la edad gestacional diagnosticada por palpación transrectal y la edad gestacional real de los animales, no obstante, se observó una disminución en la correlación entre los días 150-240. Este resultado puede ser explicado teniendo en cuenta que a partir del $5^{\circ}$ mes el feto se ubica en el piso del abdomen, lo que impide o dificulta la palpación directa del mismo o al menos algunas partes de el. Durante este tiempo, los placentomas son las principales estructuras utilizadas como referencia para determinar la edad gestacional y como ayuda adicional, la hipertrofia de la arteria uterina media, lo que necesariamente afecta la exactitud del diagnóstico.

\section{Conclusiones}

Se observó que el método de palpación transrectal del tracto reproductivo y sus anexos en la especie bovina es confiable, de excelente precisión entre los 30 y 115 días de gestación y se constituye en una herramienta indispensable para el veterinario de campo encargado de manejar los aspectos productivos y reproductivos.

\section{Referencias}

ABBITT B., BALL L., KITTO G.P., SITZMAN C.G., WILGENBURG B., RAIM L.W. 1978. Effect of three methods of palpation for pregnancy diagnosis per rectum on embryonic and fetal attrition in cows. $J$ Am Vet Med Assoc. 73:973-977.

Fricke P.M., Ricci A., Giordano J.O., Carvalho P.D. 2016. Methods for and Implementation of Pregnancy Diagnosis in Dairy Cows. Vet Clin Food Anim. 32(1):65-180.

ROMANO, J.E., THOMPSON J.A., FORREST D.W., WESTHUSIN M.E., TOMASZWESKI M.A., KRAEMERB D.C. 2006. Early pregnancy diagnosis by transrectal ultrasonography in dairy cattle. Theriogenology. 66(4):1034-1041

LÓPEZ-GATIUS F. 2005. The effect on pregnancy rate of progesterone administration after manual reduction of twin embryos in dairy cattle. J Vet Med A Physiol Pathol Clin Med. 52:199-201.

MOMONT H. 1990.Rectal palpation: safety issues. Bovine Pract. 25:122-3

OLTENACU P.A., FERGUSON J.D., LEDNOR A.J. 1990. Economic evaluation of pregnancy diagnosis in dairy cattle: a decision analysis approach. J Dairy Sci. 73(10):2826-31.

THURMOND M.C., PICANSO J.P. 1993. Fetal loss associated with palpation per rectum to diagnose pregnancy in cows. J Am Vet Med Assoc. 203(3):432-5.

VAILLANCOURT D, BIERSCHWAL CJ, OGWU D, ELMORE RG, MARTIN CE, SHARP AJ, YOUNGQUIST RS. 1979. Correlation between pregnancy diagnosis by membrane slip and embryonic mortality. J Am Vet Med Assoc. 175(5):466-8.

WARNICK LD, MOHAMMED HO, WHITE ME, ERB HN. 1995. The relationship of the interval from breeding to uterine palpation for pregnancy diagnosis with calving outcomes in holstein cows. Theriogenology. 44(6):811-25.

YOUNGQUIST RS. 2006. Pregnancy diagnosis. Current therapy in large animal theriogenology. St. Louis, MO: Elsevier Health Sciences.

ZEMANJIS R. 1966. Reproduccion animal: diagnóstico y técnicas terapéuticas. Mexico: Limusa. 